CAROLINA SHORT

DEPARTMENT OF COMPUTER SCIENCE

UNIVERSITY OF WAIKATO

HAMILTON, NUEVA ZELANDIA

CAROLINA.SHORT@WAIKATO.AC.NZ

TOMÁS GARCÍA FERRARI

DEPARTMENT OF COMPUTER SCIENCE

UNIVERSITY OF WAIKATO

HAMILTON, NUEVA ZELANDIA

TOMAS.GARCIAFERRARI@WAIKATO.AC.NZ

MARCELA QUIJANO

HfG-ARCHIV ULM

ULMER MUSEUM

ULM, ALEMANIA

M.QUIJANO@ULM.DE

\section{HfG-Archiv Ulm Online. De la realidad exclusiva a la virtualidad inclusiva}

HfG-Archiv UIm Online. From Exclusive Reality to Inclusive Virtuality

Resumen. El Archivo de la Escuela de Ulm (HfG-Archiv Ulm) comenzó a funcionar en 1987 por iniciativa de exalumnos de la misma que, tras el cierre de la Escuela (HfG - Hochschule für Gestaltung Ulm), vieron la necesidad de preservar el material sobre los proyectos producidos durante su funcionamiento entre 1953 y 1968. Se fundó como una institución pública, auspiciada por la Comuna de Ulm. Entre 1999 y 2001 se realizó un proyecto que le daría visibilidad y difusión al Archivo, mediante la creación de un sitio web. Su función era acercar al público de manera masiva a lo que fue la HfG-Ulm, sus influencias, su legado, y qué sucedía en la actualidad en relación con la historia de la misma y a sus protagonistas.

Palabras clave: archivo, HfG-Ulm, sitio web.

Abstract. The Archive of the School of Ulm (HfG-Archiv UIm) began operating in 1987 following an initiative by a group of alumni that, after the closure of the School (HfG - Hochschule für Gestaltung UIm), saw the necessity to preserve the material concerning the projects produced during its operation between 1953 and 1968. It was founded as a public institution, sponsored by the Municipality of UIm. Between 1999 and 2001 a project that would bring visibility and dissemination to the Archive was developed by creating a website. Its function was to bring towards a massive audience what was the $\mathrm{HfC}-U \mathrm{~lm}$, its influences, its legacy, and what happened today in connection with the history of the school and its protagonists.

Keywords: archive, HfG-Ulm, website.

Fecha de recepción: 16/03/2015

Fecha de aceptación: 12/04/2016

Cómo citar: Short, C., García Ferrari, T.y Quijano,

M. (2016). HfG-Archiv UIm Online. De la realidad exclusiva a la virtualidad inclusiva. RChD: creación

y pensamiento, $1(1), 13-25$.

DOI: $10.5354 / 0718-2430.2016 .44192$

Revista Chilena de Diseño,

RChD: creación y pensamiento

Universidad de Chile

2016, I(1)

http://rchd.uchile.cl 
1. En su libro el autor usa el término homestead-

ing comparando a los pioneros de América del

Norte con la apropiación de la red (internet) por parte de las empresas de medios y telefonía a principios de los noventa.

2. Winograd afirma que hay tres maneras básicas para interactuar con el mundo: conversación, manipulación y locomoción. En el diseño de computadoras, las primeras máquinas fueron pensadas desde lo conversacional, en una segunda etapa, con el uso de la metáfora del escritorio y el mouse, se introdujo la manipulación, y en una tercera fase, de la web, los usuarios empezaron a pensar en términos de locomoción, de ir a visitar un sitio yendo hacia él (Moggridge, 2007).

\section{Introducción}

El presente artículo se propone documentar la creación del website original para el Archivo de la Escuela de Ulm (HfG-Archiv UIm). El mismo fue gestado en 1999 como un proyecto que le daría visibilidad y difusión al Archivo mediante el uso de la web, un medio amplio y democrático de difusión masiva. El desafío era desarrollar un espacio fácil de navegar y de mantener, que crecería y evolucionaría con el paso del tiempo. Era un momento en el cual tener presencia en la web se había tornado como una necesidad de "tomar posesión" de un lugar -en el ciberespacio- similar al concepto de homesteading que describe Rheingold (1993), ${ }^{1}$ que en muchos casos trascendía la necesidad real de personas, instituciones y empresas de tener un website. Pero en este caso se trataba de usar la virtualidad de la web por su valor cognoscitivo (Maldonado, 1994) para dar a conocer la existencia del Archivo, sus actividades, y brindar información relativa a la HfG-Ulm.

Es interesante notar el contexto en el que se trabajó en los comienzos de este proyecto. En ese momento, en Alemania, la mayoría de las instituciones públicas recién comenzaba a plantearse la necesidad de una conexión a internet, con lo cual el acceso a los servicios de la red era muy limitado. Los websites de instituciones similares al Archivo eran muy básicos (algunas pocas páginas a modo de brochure), o bien eran inexistentes. Esto hacía muy complicada la tarea de proyectar el propio website del Archivo, sin ninguna referencia y con pocos recursos tecnológicos genuinos (ni siquiera el acceso a internet era un servicio de uso cotidiano, o era sumamente lento). En ese momento se pensó que el website para el HfG-Archiv Ulm era esperado por un gran número de personas en todo el mundo, ya que había muy poca (si no nula) información en internet sobre esta escuela que marcó un camino importante en la educación de diseñadores. Todo el material y el legado que el Archivo poseía podía ser apreciado únicamente si se visitaba personalmente ese lugar físico en la ciudad de Ulm. Hasta el año 2000 solo un pequeño porcentaje de material acerca de la Hfc se conocía a través de publicaciones, que muchas quedaba circunscrito a determinadas regiones, ya sea por su distribución o por el idioma en el que se habían publicado. Para muchos estudiantes que estaban siendo educados con el Modelo de Ulm, como es el caso de Latinoamérica, la Hfc era completamente desconocida. Sin embargo, para los iniciadores de este website era muy claro. Tomando la metáfora de locomoción de Terry Winograd, en la que explica que a medida que la internet se hacía más popular y se inclinaba hacia el uso de la web, el uso de las computadoras se transformó en un medio por el cual uno podía moverse e ir a distintos lugares, la idea era que el público pudiera "visitar" el archivo desde cualquier lugar del mundo. ${ }^{2}$ Asimismo, presentar al Archivo en la web era darle una telepresencia, como la que describe Maldonado en Lo real y lo virtual:

Es evidente que meterse en una realidad virtual no es igual que hacerlo en una realidad real. Pero teóricamente nada impide aceptar que nuestra interacción con la primera nos ayuda a adquirir nuevos conocimientos sobre la segunda. Por más que haya grandes discontinuidades, hay que admitir empero que entre las dos realidades no faltan continuidades igualmente importantes. (Maldonado, 1994) 
Curiosamente, el equipo de trabajo que proyectó y realizó el nuevo sitio fue integrado por personas originarias de Alemania, Colombia y Argentina, fiel al ejemplo de la internacionalidad de la Escuela de UIm. En 1998 Marcela Quijano, curadora del HfG-Archiv, Carolina Short y Tomás García Ferrari fueron becarios en la Akademie Schloss Solitude, ${ }^{3}$ en Stuttgart. Los tres fueron escogidos por Gui Bonsiepe, jurado de la institución para el área de Diseño en el período 1997-1998. Esta casualidad y el hecho de que Bonsiepe como egresado de la Escuela de Ulm tuviera un papel importante en la difusión de los nuevos medios, generó la posibilidad de que se conformara un equipo internacional, permitiendo que la realización del website no se hiciera con alguna oficina/ agencia web local. La mayor parte del trabajo se llevó a cabo entre Ulm y Buenos Aires, Ulm y Nueva Zelanda, haciendo palpables las posibilidades de la web, y según lo afirmaba Bonsiepe: "La computadora no es una herramienta de trabajo individual sino un instrumento social que abre nuevas formas de cooperación". Ya a fines de los años noventa la internet era un medio de comunicación muy poderoso que permitía un contacto bidireccional fluido, no solo entre personas de una institución (como las universidades) sino con el resto del mundo. "No soy pesimista, si no, no podría ser proyectista. Me alienta lo digital, que se abran espacios de comunicación y participación a través de la red. Y el diseñador como responsable de introducir y hacer manejable la tecnología en la vida cotidiana de las personas" (Cambariere, 2005). Desde lo conceptual, el objetivo original del desarrollo de la World Wide Web fue facilitar el flujo de la información entre científicos mediante un proceso de vinculación de distintos artículos con sus respectivas referencias, permitiendo - a través del uso de un sistema de hipertexto- que los usuarios consulten dichas referencias a medida que fueran leyendo. Sin embargo, el objetivo original de la web, además de hacer accesible los datos, fue también hacer factible la posibilidad de publicar información por todos y cada uno de sus usuarios, algo que comenzó a suceder con el advenimiento de los weblogs y las redes sociales (García Ferrari, 2009).

\section{El Archivo de la Escuela de Ulm y la www en el momento de comenzar el proyecto}

El Archivo de la Escuela de Ulm (HfG-Archiv Ulm) fue abierto por el consejo municipal de la ciudad de UIm en 1987, por iniciativa de exalumnos de la misma. Tras el cierre de la Hfc el material sobre los proyectos producidos durante su funcionamiento quedó a disposición y con la creación del Archivo, se sumaron donaciones y legados, como los de los exprofesores Otl Aicher, Walter Zeischegg, Tom Gonda, entre otros. La función del mismo es preservar el material histórico y organizar exhibiciones y eventos en forma continua y regular, editar libros, y otras actividades de difusión en relación con la institución y sus integrantes.

A fin de comprender lo que significaba proyectar un website en ese momento histórico, se describirá cómo era el contexto en el cual estaría inscrito el proyecto del Archivo.

En un artículo publicado en el libro Gebrauch und Gebräuchlichkeiten(Aicher \& Rinker, 2000), que se editó con motivo del segundo simposio organizado en Rotis por el Archivo y Rotis Forum, se señalaba lo siguiente: "La importancia de la internet se basa en diversos factores. Primero que todo, está disponible mundialmente (con algunas excepciones, y el hecho que hasta el momento solo el $3 \%$ de la población global tiene acceso a internet). Esto
3. Institución alemana que ofrece becas para artistas y científicos en diferentes disciplinas ("Akademie Schloss Solitude", s.f.) 
4. Del latín "Verba volant scripta manent".

5. Para tener un punto de comparación, en junio de 2014 había un número estimado de 3.035 millones de usuarios, el equivalente a $42,3 \%$ de la población mundial ("Internet Growth Statistics the Global Village Online", s.f.). constituye una gran diferencia con otros medios. A su vez, otorga la posibilidad de una comunicación en dos direcciones (en este caso, el sentido de "emisario" y "receptor" está aún más borroneado) a un costo muy bajo. No es complicado (en términos prácticos ni económicos) crear y distribuir cualquier tipo de información instantáneamente y distribuirla a todo el mundo. La democratización del proceso de publicación ya ha comenzado y hoy está - más o menos - en el mismo nivel la información publicada por CNN y por un estudiante de una escuela de Sudamérica. Todo este proceso de hacer accesible la información está siendo más fácil, rápido y barato. Por otra parte, está cambiando la economía en todo el mundo. Los sistemas de comercio electrónico (e-commerce) recién se encuentran en sus inicios y hay mucho por venir en un futuro. En muchos sentidos ha creado una revolución comparable con la imprenta de Gutenberg, 500 años atrás. Pero desde el punto de vista del diseño, el desarrollo de la presencia de una compañía o una institución en la internet es mucho más complejo que diseñar unas pocas pantallas. Implica tomar decisiones de distinto tipo: de comunicación, tecnológicas y de diseño. La creación de un website involucra áreas como marketing, sistemas y diseño, a fin de conformar un equipo multidisciplinario" (García Ferrari y Short, 2001). Esta cita da cuenta de cómo era en aquel momento pensar en un proyecto para la web. El proyecto debía tomar en cuenta que sería creado con el objetivo de permanecer en el tiempo ("lo escrito permanece"), ${ }^{4}$ a diferencia de una pieza impresa en la cual la duración tiene una fecha de impresión (y muchas veces un vencimiento). La idea, común a muchos websites, era establecer contacto con el público, dar a conocer información relativa a su funcionamiento (horarios, tiempos de visita, cómo llegar, entre otras), difusión de los contenidos del Archivo y de lo que fuera la Escuela de Ulm en sí, promoción de eventos y publicaciones relacionadas con la misma.

El mapa original de la primera versión del website refleja las distintas áreas que serían tomadas en cuenta y de qué manera se organizarían los contenidos y se interconectarían entre sí las distintas secciones y páginas. El desarrollo de la World Wide Web puede ser entendido en diferentes eras de acuerdo a los esquemas tecnológicos utilizados. En un primer período (comprendido entre 1991 y 1999) se plantean los sitios web como red de documentos HTML, estáticos, en tanto son creados una única vez y almacenados en servidores desde los cuales pueden ser obtenidos; en un segundo período (entre el año 2000 y 2009) se piensa en estructuras creadas sobre bases de datos, donde los documentos se generan de manera dinámica en los servidores integrando plantillas y registros en bases de datos relacionales (con el predominio de una base tecnológica denominada LAMP, acrónimo de Linux, Apache, MySQI y PHP); y en un tercer período (que comienza alrededor del año 2010), donde se empieza a pensar los sitios web como un flujo continuo de eventos (Driscoll, 2011). Desde este punto de vista, este proyecto fue pensado dentro del primer período, como una red de documentos HTML estáticos interconectados entre sí.

En el año en que se comenzó a planificar el website (1999) muchos de los servicios que hoy en día utilizamos cotidianamente no existían o apenas comenzaban a existir. A fines de dicho año, la web contaba con aproximadamente 250 millones de usuarios, el equivalente al 4,1\% de la población mundial.5 Por citar algunos ejemplos ilustrativos, el buscador Google había sido creado apenas un año antes. El concepto de descargar música en formato mp3 empezó dicho año con una compañía que ya no existe: Napster. En el 


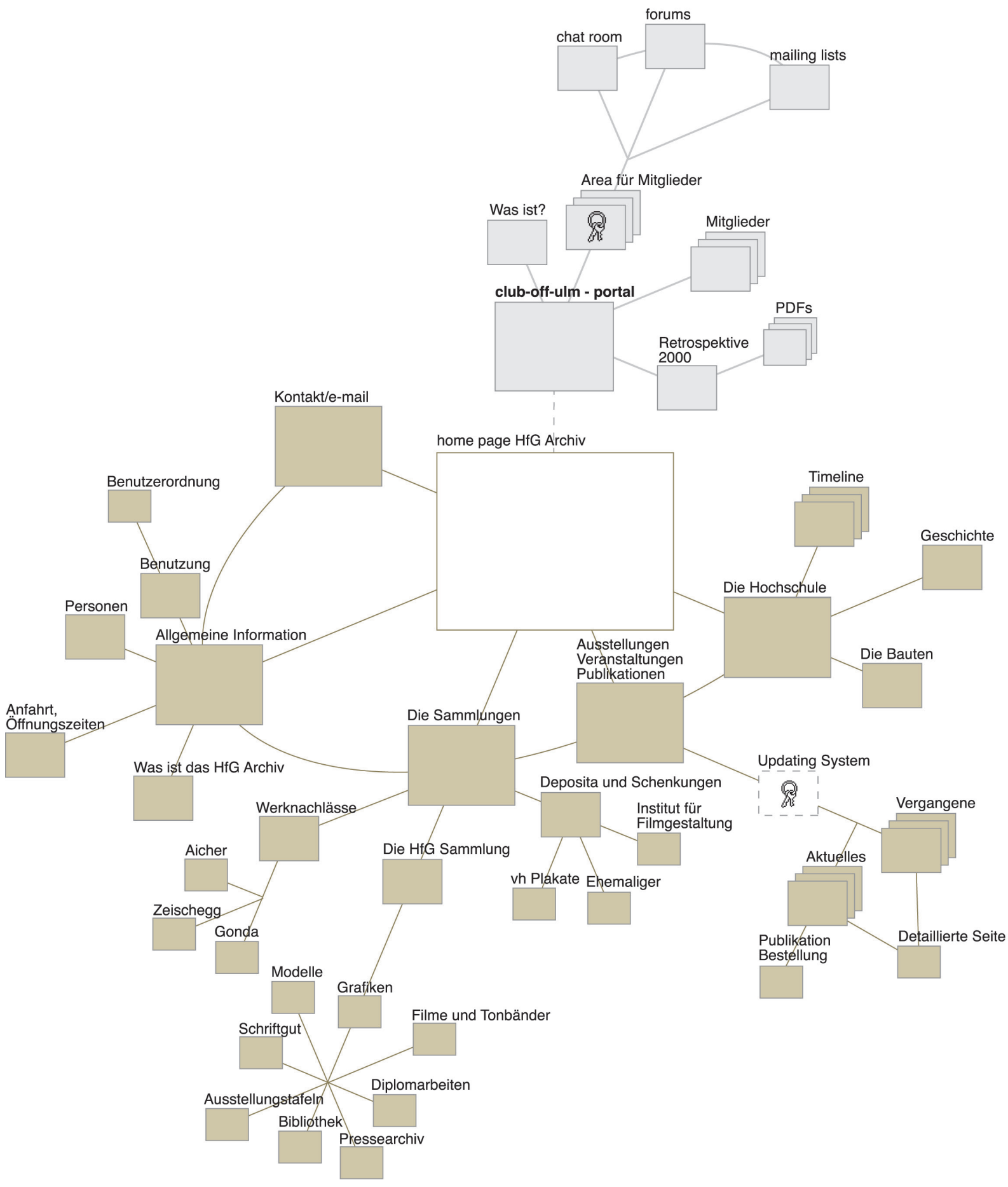

Figura 1. Mapa del sitio en su versión original, después no se llevó a cabo el portal club-off-ulm, y algunas de las secciones cambiaron sus nombres, otras se subsumieron, y se agregaron a medida que se fue definiendo el proyecto original.

Fuente: Gebrauch und Gebräuchlichkeiten (Aicher \& Rinker, 2000). 
6. Nicholas Negroponte (1995), definía que los átomos forman parte de objetos físicos y tangible como CD, libros y cartas. La información digital, por su parte, está compuesta por bits, la unidad mínima de información empleada en informática, en cualquier dispositivo digital. El autor escribía que todas las formas de información que estaban hechas de átomos eventualmente serían convertidas a bits. De hecho es lo que ha sucedido en las últimas décadas con la digitalización de muchas formas de comunicación. año 2000 tiene lugar el boom de las empresas punto.com; es un momento en que muchas compañías relacionadas con los medios digitales se hacían públicas en el mercado financiero, principalmente en Estados Unidos. El usuario de internet pasaba conectado un promedio de 83 minutos diarios ("World Wide Web Timeline", 2014). En enero de 2001 Jimmy Wales y Larry Sanger lanzan Wikipedia, y los primeros usuarios escriben cerca de 20 mil artículos durante ese año de inicio. La enciclopedia online es un recurso que hoy en día nos parece tan natural para consultar, como muchos otros servicios tales como hablar mediante videoconferencias, publicar nuestro perfil profesional y hacer conexiones con colegas -en el año 2003 comienzan a existir Skype y Linkedin-, o mirar y publicar videos - YouTube se funda en febrero de 2005 y el primer video lo publica uno de sus fundadores, Jawed Karim, el 23 de abril de dicho año. Los teléfonos inteligentes (teléfonos móviles que utilizan un sistema operativo) existen desde fines de los 9o, pero se comenzaron a popularizar a partir del 2007, permitiendo utilizar la tecnología touch screen y contar con posibilidades de uso similares a los del PC. Estos son algunos de los hitos que se proponen como ejemplo para dar un contexto; también se podría hablar del crecimiento/evolución de las conexiones a internet y de los dispositivos utilizados para acceder y utilizar la misma.

\section{Desarrollo y planificación del website}

Teniendo en cuenta los objetivos que tendría que cumplir el proyecto, había que elegir los contenidos del mismo. El proceso de selección era bastante complicado, ya que no se contaba con ningún material digitalizado, con lo cual había que tomar en cuenta de qué manera se iba a traducir la información "de átomos a bits". ${ }^{6}$ El Archivo tampoco contaba con un área específica de comunicación que tuviera lineamientos o material preestablecido como promocional o de difusión, sino más bien algunas piezas aisladas, en general referidas a exhibiciones o eventos particulares. La tarea era definir cómo acompañar al Archivo desde la Web, usada como plataforma para difusión de información relativa al Archivo, información que a su vez debió ser creada desde fojas cero y adaptada para ser publicada en el medio digital. Los contenidos se organizaron en distintas secciones según el tipo de información y a qué se referían:

- Información institucional

Datos concretos para visitar el lugar, personal a cargo

- La muestra o colección del Archivo

Detalle de los distintos aspectos en profundidad

- Exhibiciones

Organizadas/relacionadas con el Archivo o la HfG-Ulm

- Eventos

Organizados/relacionados con el Archivo o la HfG-Ulm

- Publicaciones

Actuales, pasadas, externas y bibliografía

- La Escuela de UIm

Historia, edificio, línea de tiempo

El listado muestra cuál era el primer nivel del website, que estaba marcado por las páginas de apertura de cada sección, con el fondo del color de la misma. En este nivel se presentaban las generalidades de cada sección y se anticipaban los contenidos que se darían en un segundo nivel. Este último, brindaba más expli- 
caciones de cada tema y tenía en algunos casos, a su vez, acceso a un tercer nivel en el cual se desarrollaba con más detalle. Desde la página inicial (home page) se tenía acceso directo a ciertas páginas con la información más destacada. Un punto a tener en cuenta al realizar la calificación y la organización de la información era los intereses diversos que podían tener los usuarios al visitar el website. El acceso estaría definido por el uso, planteando distintos planos de información. Algunos simplemente querrían saber cómo llegar al lugar, para lo cual necesitaban la información concreta de la dirección y los horarios. Otros, llegarían al website con la finalidad de encontrar información sobre la HfG-Ulm, datos históricos y concretos sobre el período en el que funcionó. Para usuarios en busca de información más específica, estarían las secciones como "La muestra" o "Publicaciones", que brindaban datos sobre material en particular para ser consultado con mayor profundidad. A su vez, secciones más actuales como "Exhibiciones" o "Eventos", brindarían información al día de actividades relacionadas con la $\mathrm{HfG}-U \mathrm{Im}$ que sucedían en el marco de la actualidad. El programa visual del website se realizó tomando como base las pautas gráficas y visuales que venían usando en el Archivo, y en combinación con el programa visual de la ciudad de Ulm. Se buscaba como objetivo lograr un buen balance entre material gráfico y textos, el primero como apoyo fundamental en la transmisión de la información, tomado no desde lo puramente estético, sino desde su valor semántico. El concepto visual del proyecto se focalizó en el diseño funcional y consistente de la información. Era claro que el contenido tenía que brillar por sí mismo y, como ese era uno de los objetivos principales, se intentó mantener la interfaz visual lo más neutral posible, haciéndola simple y fácil de utilizar.

El layout se definió a partir de las seis secciones que integraban el contenido. Se diseñó una grilla de seis columnas, que se podría utilizar de manera flexible, acomodándose a las necesidades de las diferentes secciones: texto e imágenes, listados, descripción de publicaciones, etc.

Se definió el uso de un color para cada sección, a fin de darles una identidad definida, y se determinó que abrirían con una portada utilizando un póster de la colección del Archivo como elemento gráfico. Dicha aplicación cromática tendría un rol menor, sería aplicado únicamente en el banner superior, el menú, y algunos textos en cuerpos pequeños como los epígrafes, de modo de cohesionar esta pauta de identificación sin interferir con el resto del contenido. Las fotografías, color y gráficos darían otra nota de color al proyecto. La navegación tenía múltiples opciones, era redundante en el sentido de poder seleccionar la misma información de diferentes maneras, permitiendo recorridos diferenciados. Ambos elementos estarían presentes en todas las páginas y el menú, al ser desplegable (contextual), habilitaba la navegación directa a cualquier página del sitio desde cualquier punto. Además, se seleccionó este tipo de menú porque economiza espacio al tomar un pequeño porcentaje de la pantalla. Mediante el uso del banner superior el usuario podía ir a la home, y desde allí este funcionaba como un vínculo al sitio de la ciudad de UIm, de manera de mantener una relación con aquella, reflejando la relación existente desde lo institucional. A su vez, todo el sitio contaba con breadcrumbs $s^{7}$ que funcionaban como orientación, como un mapa, dándole al usuario la noción del lugar en el que se encontraba dentro del sitio. En la sección de la colección, que contaba con material gráfico fotográfico, la navegación se estableció mediante el uso de thumbnails, estampillas que anticipaban lo que se podía visualizar con mayor detalle, y en páginas con
7. "Miga de pan" (breadcrumb en inglés) es una técnica de navegación usada en interfaces gráficas -incluidas las páginas de la web- consistente en una línea de texto que indica el recorrido realizado y contiene vínculos que permiten regresar. El usuario puede ver así su ubicación en directorios y subdirectorios, y navegar a través de ellos. El término tiene su origen en el cuento clásico Hansel y Gretel de la colección de los Hermanos Grimm. 

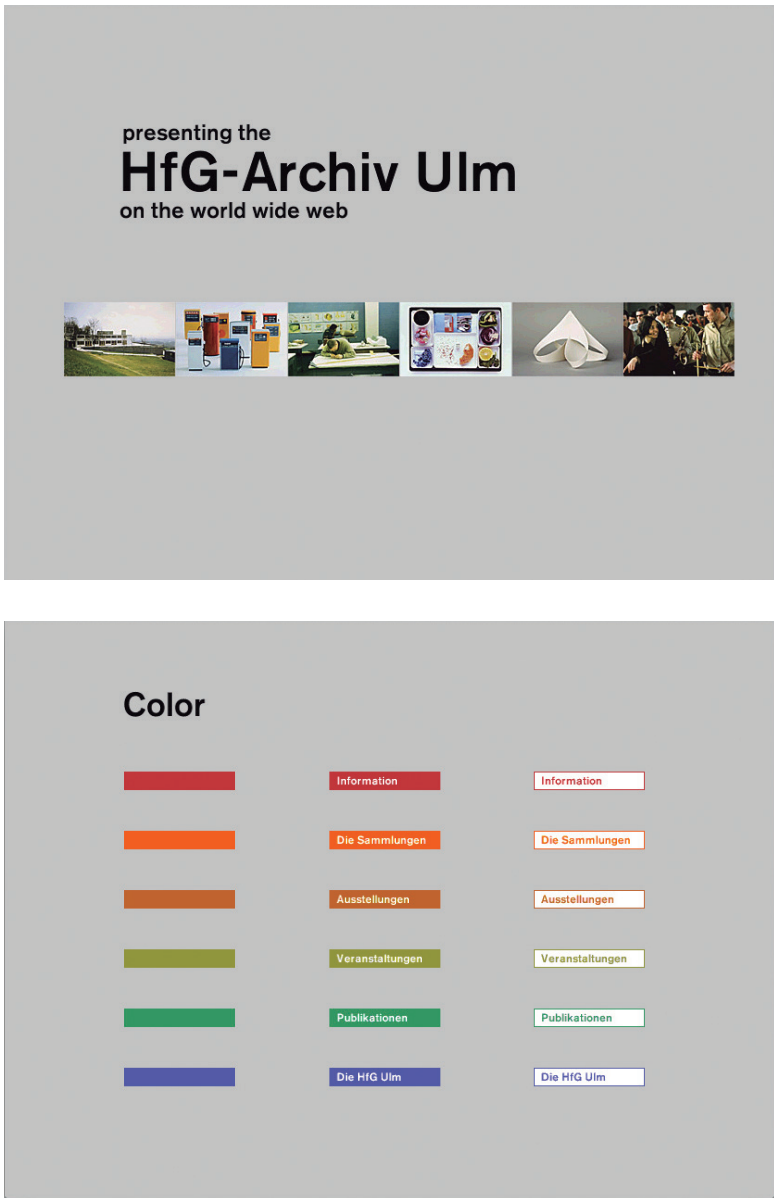

\section{Layout}

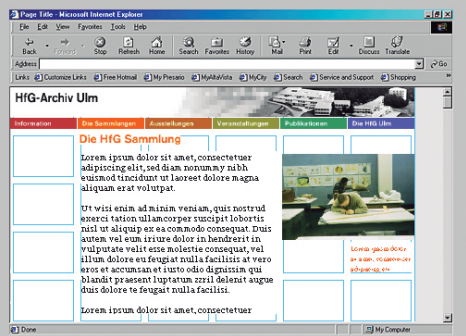

\section{Structure and content}

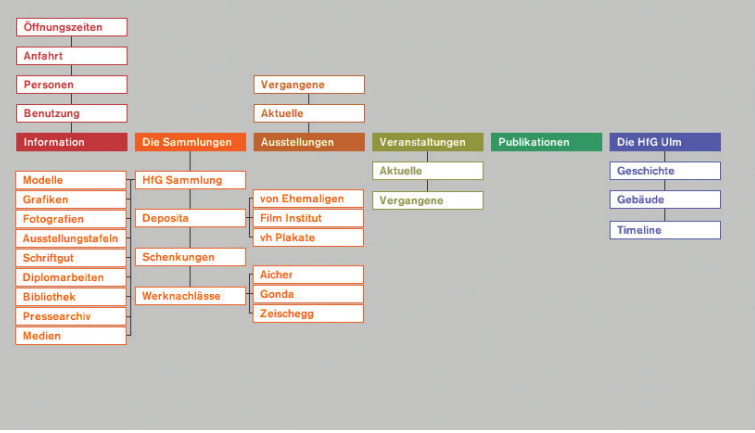

Typography

Berthold Akzidenz Grotesk

\section{abcdefghijklm nopqrstuvwxyz ABCDEFGHIJ KLMNOPQRST UVWXYZ 1234567890 !@\#\$\%^ $\boldsymbol{\&}^{*}() ?$}

ssued by Berthold in 1896, Akzidenz Grotesk is the forerunner of Helvetica. The name is derived from the nineteenth-century word for in sans seri, Grotesk, and from used by trade printers, Akzidenz schrift.

\section{Layout}

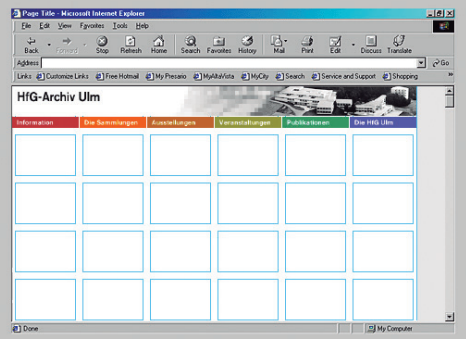

Figura 2. Presentación proyecto ante el Club-off-ulm (Zurich, octubre 2001). Fuente: Archivo de los autores. 
más texto, como las de historia, se agregó un paginado como menú secundario que permitía ver de antemano cuántas páginas contenía esa sección y navegar entre ellas.

La tipografía elegida fue Verdana, una fuente tipográfica diseñada especialmente para ser usada en la pantalla, que contaba con la legibilidad óptima, y a la vez, al ser una sans serif, representaba el espíritu de la HfG, que durante los años de su funcionamiento utilizara sans serif tales como Helvetica o Akzidenz Grotesque. La Verdana, diseñada en 1996 por el tipógrafo inglés Matthew Carter para Microsoft, debido a su popularidad fue en su momento la "Helvetica de la www" (García Ferrari, 1997), y al haber sido concebida desde la grilla de pixeles no solo la forma de las letras sino los pesos tipográficos y el interletrado estaban optimizados para la lectura en pantalla. La elección se realizó teniendo en cuenta, como dice Bonsiepe, que "El diseño tipográfico es mucho más que un servicio marginal y debe ser valorado como un elemento central del lenguaje [...] no debe ser considerado como un accesorio, sino un dominio que hace que los textos sean reconocibles como objetos estéticos. Si el lenguaje hace visible y comprensible la realidad, a su vez, la tipografía hace visible y comprensible el lenguaje como texto, siendo esta, por lo tanto, básica para la comprensión" (Bonsiepe, 1993).

Respecto de la selección tipográfica, hay una anécdota interesante que tiene su origen en la presentación del proyecto, realizada ante el Club-off-ulm. ${ }^{8}$ Dicha reunión contó con la presencia de exalumnos y profesores de la Hfc. En una primera etapa, se había establecido que para el sitio se utilizaría una tipografía sans serif para títulos y subtítulos y una tipografía con serif, para los textos, debido a una limitación tecnológica del momento, que impedía utilizar la familia Akzidenz Grotesk, que entendíamos era la utilizada por la escuela. Esta propuesta tuvo un gran rechazo por parte de la audiencia, que consideraba que la tipografía de la HfG-Ulm era únicamente sans serif. Evidencia de esto se puede hallar en pósters, la revista ulm, y otros materiales impresos de la Escuela. Entre los presentes, se encontraban exalumnos que habían trabajado en el Departamento de Comunicación Visual de la Hfc y explicaron que en realidad se utilizaba la tipografía que había en el taller (en aquel momento, tipos móviles usados en una impresora). Y que los tipos con los que contaban no eran únicamente Akzidenz Grotesk, una sans serif precedente a la Helvetica, desarrollada por la fundidora alemana Berthold, sino también en algunos casos, la misma Helvetica, desarrollada por la fundidora suiza Haas. Con lo cual, el mito de que la elección tipográfica ulmiana fuese estrictamente una decisión conceptual se relativizaba bastante.

Bonsiepe escribía: “Antes de la difusión de la computadora personal, las posibilidades de diferenciación tipográfica se limitaban por motivos tecnológicos y económicos. Hoy, en cambio, el gráfico puede experimentar en tiempo real y prever en un juego virtual las consecuencias de sus decisiones con un feedback directo. Es probable que una causa tecnológica determine el surgimiento de variedades tipográficas y del pluralismo en la proyectación que podemos observar hoy en día". Un poco este texto acompaña la decisión de optar por una sans serif, que de alguna manera acompañe la identidad de la HfG-Ulm, tal como se solicitaba en la presentación mencionada, pero que sea una tipografía diseñada desde la pantalla, como la Verdana. El website en sus inicios estaba publicado solamente en alemán, y en 2003 se realizó una traducción al inglés, y un ajuste del mismo al agregado de esta traducción. Unos años más tarde, con el advenimiento de los weblogs, y con motivo
8. El Club-off-Ulm es la fundación de exalumnos de la HfG-Ulm que financió parte del website <http://www.club-off-ulm.de>. 

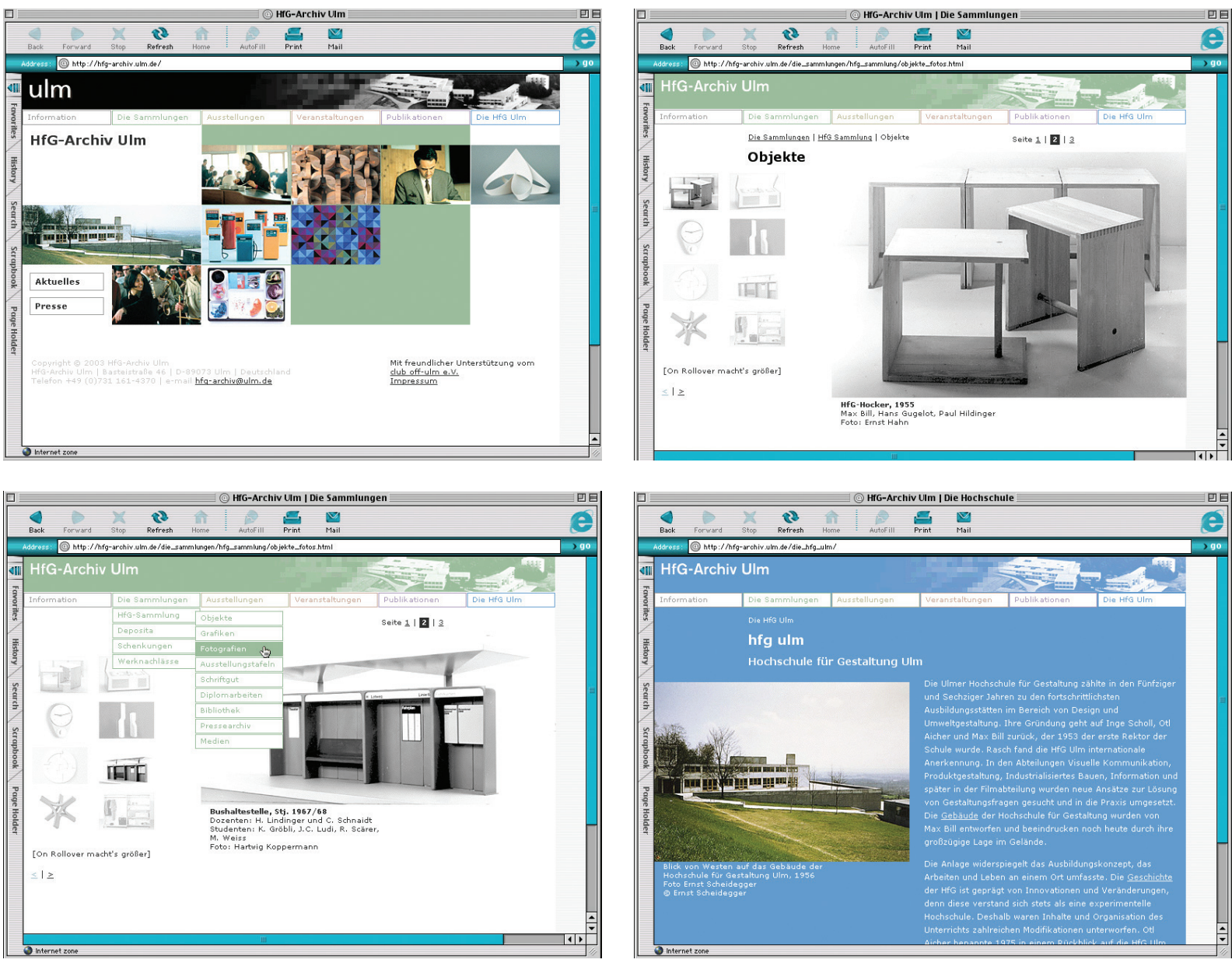

Figura 3. Páginas del sitio en su primera versión. Fuente: Archivo de los autores.

9. Una de las propuestas es la de incluirlo en la plataforma online de la ciudad de UIm, tal como el caso del Museo de la Ciudad de UIm (UImer Museum), <http://www.ulm.de/kultur_tourismus/museen_und_bildende_kunst/aktuelles.109222.3076,3963,3850,3586,109222.htm>. de una necesidad concreta del Archivo de mantener al público al tanto del plan de mudar el Archivo al edificio original de la HfG-Ulm, se diseña un blog ("hfg-archiv blog", 2009).

La función del mismo sería abrir un canal de comunicación proponiendo temas para que el público pudiera dejar comentarios. Contaba con posts propios del Archivo y de algunos colaboradores, y se publicaron artículos hasta 2012, solo en idioma alemán (Short, 2009). A partir de esa fecha el Archivo decidió el uso de las redes sociales como modo de establecer un contacto más fluido con sus seguidores.

El proceso de diseño y desarrollo del website duró unos 36 meses y en él trabajo un equipo de dos diseñadores gráficos especializados en la web como estudio independiente, y un equipo interno del Archivo.

- Dirección del proyecto Marcela Quijano

- Textos

Marcela Quijano, Dr. Dagmar Rinker y Tanja Wagner

- Colaboración:

Dr. Martin Mäntele, Michaela Gleinser, Dra. Brigitte Hausmann, María Victoria Pérez Arias.

- Diseño y desarrollo

(bi)gital» (Carolina Short y Tomás García Ferrari) 

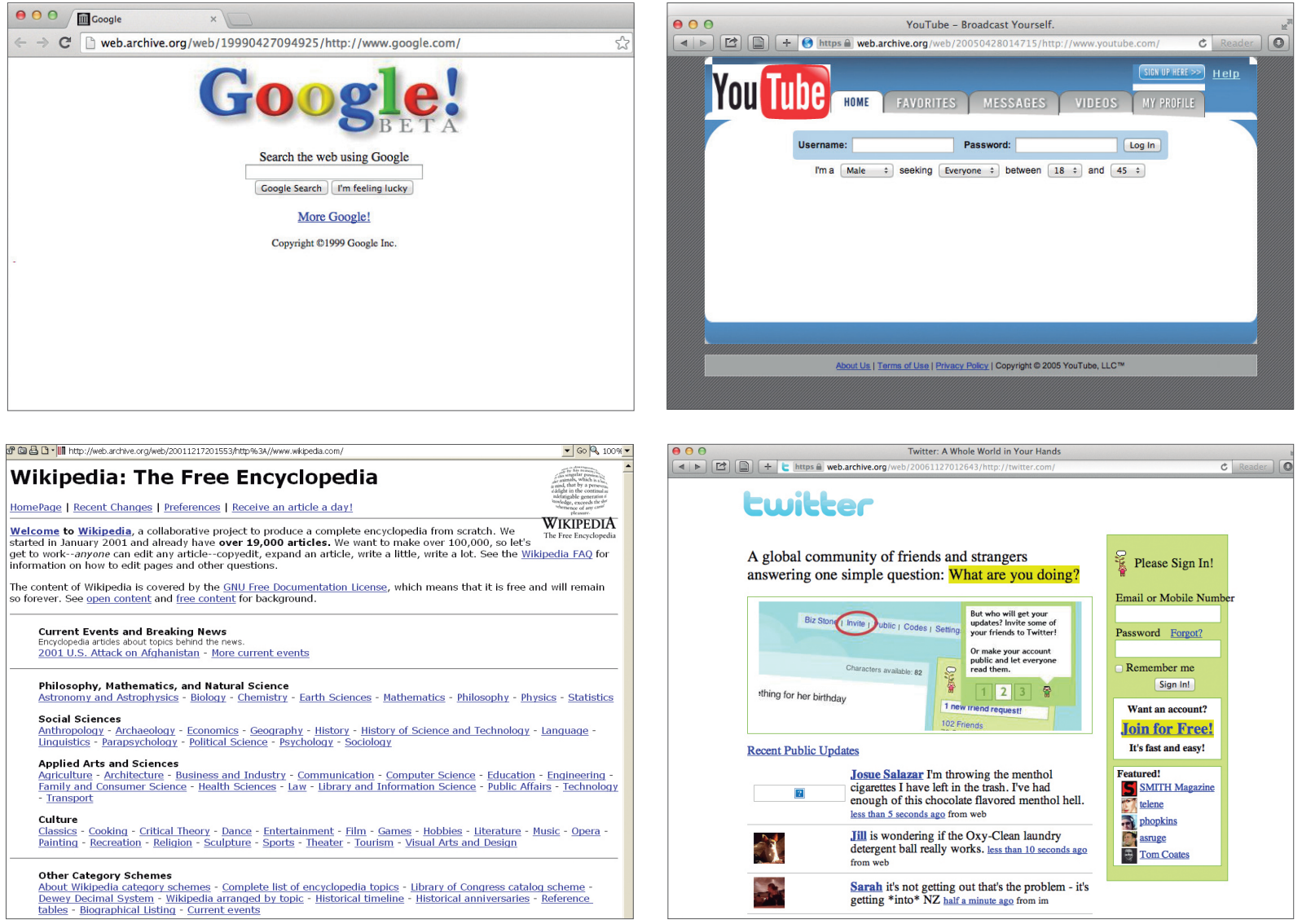

\section{Conclusión}

A pesar de que el website ha quedado detenido en el tiempo en cuanto a su diseño y su funcionalidad (en el sentido de no ser autogestionable como lo son la mayoría de los websites en su categoría), lo importante es que resulta un instrumento de alto valor comunicacional para el archivo. Seguramente la pregunta que se puede formular es por qué después de tantos años no ha sido actualizado. Si bien han existido algunas posibilidades de renovación, ${ }^{9}$ las propuestas de cambio no resultaron superadoras desde el valor comunicacional sino solamente desde la forma o el aspecto visual. Una actualización requiere de una inversión que muchas veces no se prioriza porque es complicado medir el valor (en términos económicos) de un instrumento de comunicación de su tipo.

El website del HfG-Archiv se ha convertido, con los años, en un archivo, y sus componentes virtuales son como extensiones de los objetos (tanto tangibles como intangibles) contenidos en el Archivo. Wofgang Ernst hace referencia a la internet como archivo en su libro Digital Memory and the Archive (Ernst \& Parikka, 2013), y en esa dirección no cabe duda que una actualización que permita ampliar el website, sus contenidos y sus funcionalidades va a darle al Archivo aún una versión mucho más rica para cumplir con su misión de coleccionar y conservar, y ser, a la vez, un instrumento de investigación, de enseñanza y divulgación en relación a la Escuela de UIm. Constituye un archivo del Archivo, además, no solo por la digitalización de mucha de la información que se tradujo a fin de ser publicada, sino porque
Figura 4. Versión histórica de sitios existentes, icónicos en su tipo: Google (1999), YouTube (2005), Wikipedia (2001) y Twitter (fines de 2006). Muchos de ellos son contemporáneos con el website del HfG-Archiv UIm.

Fuente: Internet Archive Wayback Machine, <https://archive.org/web/>. 
10. Si bien Maldonado hacía referencia a ejem-

plos en el campo de la investigación experimen-

tal de las ciencias principalmente, se podría apli-

car a cualquier pieza de software. "Precisamente

por esta naturaleza sincrética que presentan,

los modelos informáticos pueden ofrecer a la

investigación científica y a la planificación, en

todos los campos, posibilidades que nunca hubo

en el pasado. En lugar del tradicional modo de

abordar los problemas, es decir, recorriendo un

largo y fatigoso itinerario de pruebas y errores,

ahora se emplea un método en el que pruebas

y errores piden una inversión de tiempo y de

recursos sustancialmente reducida. Pruebas $y$

errores se dan ahora en el espacio de una reali-

dad eidomática, cuya interpretación hace fluida e

inmediata nuestra relación de experiencia con el

problema que queremos analizar y eventualmen-

te resolver" (Maldonado, 1994). al haber operado como medio de comunicación durante estos 15 años de su existencia, termina siendo un archivo de actividades, eventos, publicaciones del Archivo mismo, como una bitácora que almacena los últimos años de actividad de la institución. La web no fue pensada específicamente para ello, pero finalmente funciona como un medio de conservación y difusión de información. Sumado a eso, el acceso prácticamente universal que posee hace que se pueda llegar a un lugar específico, físico, en la ciudad de Ulm, en Alemania, cumpliendo con la metáfora de locomoción que propone Winograd. El computador, con la emergencia de la internet y sus aplicaciones, dejó de ser una máquina con la que el hombre realiza una tarea específica para convertirse en una máquina para comunicar todo tipo de información de distintos medios, con diferentes capas estructurales y de interacción. Se habla de navegar de un lugar a otro de la Web, tocar páginas, y seguir vínculos. Son metáforas de locomoción espacial que nos comprometen, nos involucran. Nos abren nuevas formas de pensar, de aprender, de conocer, de hacer (Winograd, 1997).

Hace más de 20 años Maldonado proponía que la modelística virtual estaba demostrando ser un "vigoroso medio de conocimiento". Un website se podría definir, en términos de este autor, como un modelo informático, un "eficaz dispositivo de simulación observadora" que permiten una observación más completa y más articulada. ${ }^{10}$

La proyección de este website debería ser la de ampliar aún más lo que está publicado, y de aprovechar las posibilidades tecnológicas actuales para mejorar la calidad del material digitalizado, ya que fue pensado para otro momento de la Web, con un ancho de banda limitado y para un tamaño de pantalla que estaba determinado por los computadores de fines de los noventa.

Para mantener y hacer crecer un proyecto en la web es imprescindible contar con recursos genuinos, ya que actualizarlo desde el contenido y desde el valor comunicacional con sus visitantes es todo un trabajo en sí mismo. Un proyecto que no se actualiza en varios años luce viejo en un medio como la web, que es altamente dinámico, y cuyo cambio es constante, acompañando el paso de los avances tecnológicos de los dispositivos que se utilizan como soporte. En este proyecto en particular se da el caso que ha sobrevivido a las diferentes actualizaciones tanto de software como de hardware (versiones de navegadores y dispositivos para navegar por la www). 


\section{Bibliografía}

Aicher, F. \& Rinker, D. (2000). Gebrauch und Gebräuchlichkeiten. Vom Umgang mit den Dingen und ihrer Gestalt. Ein Symposion in Rotis. Munchen: Christian Pixis.

Akademie Schloss Solitude. Akademie-solitude.de.

Recuperado de http://www.akademie-solitude.de/en/ institution/guiding-principles/.

Bonsiepe, G. (1993). Del objeto a la interfase. Buenos Aires: Ediciones Infinito.

Cambariere, L. (2005). Una charla de diseño. Página/12 :: m2. Recuperado de http://www.pagina12.com.ar/ diario/suplementos/m2/10-814-2005-11-12.html.

Cantz, H. (2013). Ulmer Modelle - Modelle nach Ulm: Ulm School of Design 1953-1968 (2003). Ostfildern: Hatje Cantz.

Driscoll, M. (2011). Node.js and the JavaScript Age. Metamarkets. Recuperado de https://metamarkets. com/2011/node-js-and-the-javascript-age/.

Ernst, W. \& Parikka, J. (2013). Digital memory and the archive. Minneapolis, MN: University of Minnesota Press.

García Ferrari, T. (1997). Tipografía Verdana. Tipográfica, (34). García Ferrari, T. (2008). Diseño y weblogs. Un estudio de los factores de diseño determinantes para el crecimiento de la blogósfera. Recuperado de http://bigital.com/ castellano/files/2009/o1/diseno-y-weblogs-tesinatomas-garcia-ferrari.pdf (22 de Enero de 2015).

García Ferrari, T. \& Short, C. (2000). Designing the net. En F. Aicher \& R. Dagmar, Gebrauch und Gebräuchlichkeiten. Vom Umgang mit den Dingen und ihrer Gestalt. Ein Symposion in Rotis (1st ed.). Munchen: Christian Pixis.

HfG-Archiv Blog. (2009). HfG-Archiv.Ulm.de. Recuperado de http://www.hfg-archiv.ulm.de/blog/ (8 de Octubre de 2014).

HfG-Archiv Ulm. (2001). HfG-Archiv.Ulm.de. Recuperado de http://hfg-archiv.ulm.de/ (8 de octubre de 2014).

HfG-Ulm - Club off Ulm. Club-off-Ulm.de. Recuperado de http://www.club-off-ulm.de.

How 20 popular websites looked when they launched. (2009). Telegraph.co.uk. Recuperado de http://www. telegraph.co.uk/technology/6125914/How-20popular-websites-looked-when-they-launched.html (22 de Enero de 2015).

Internet Growth Statistics - the Global Village Online. Internetworldstats.com. Recuperado de http://www. internetworldstats.com/emarketing.htm.

Maldonado, T. (1994). Lo real y lo virtual. Barcelona: Gedisa. Moggridge, B. (2007). Designing interactions. Cambridge, Mass.: MIT Press.

Negroponte, N. (1995). Ser digital. Buenos Aires: Editorial Atlántida.
Rheingold, H. (1993). The Virtual Community: Homesteading on the Electronic Frontier. Reading, Mass.: Addison-Wesley.

Short, C. (2009). El blog del HfG-Archiv Ulm (archivo de la escuela de ulm) | (bi)gital». Bigital.com. Recuperado de http://bigital.com/castellano/2009/11/el-blog-delhfg-archiv-ulm-archivo-de-la-escuela-de-ulm/.

Short, C. (2011). HfG-Archiv Ulm: volviendo a casa | (bi) gital». Bigital.com. Recuperado de http://bigital.com/ castellano/2011/11/hfg-archiv-ulm-volviendo-a-casa/.

World Wide Web Timeline. (2014). Pew Research Center: Internet, Science of Tech. Recuperado de http://www. pewinternet.org/2014/03/11/world-wide-webtimeline/ (15 de Diciembre de 2014).

Winograd, T. (1997). Interspace and an every-citizen interface to the national information infrastructure. NATL Academy Press, 260-264. 